05

\title{
Перенос и пленение резонансного излучения в двухуровневой системе
}

\author{
(С В.М. Ямщиков, В.Г. Рогачев, Е.А. Кудряшов, Г.Н. Качалин \\ ФГУП „РФЯЦ-ВНИИЭФ“, \\ 607188 Саров, Нижегородская обл., Россия \\ e-mail: oefimova@otd13.vniief.ru
}

Поступила в редакцию 25.11.2019 г.

В окончательной редакции 25.03.2020 г.

Принята к публикации 06.04.2020 г.

\begin{abstract}
Приведено теоретическое исследование кинетики заселенности двухуровневой системы с учетом переноса спонтанного излучения при непрерывной оптической накачке. Показано, что в случае, когда длина пробега фотона в резонансно поглощающей среде меньше или сравнима с характерным размером этой среды, спонтанное излучение может быть дополнительным источником накачки. При рассмотрении влияния вклада спонтанных фотонов в кинетику уровней использовались два подхода. Первый подход заключается в решении уравнения переноса излучения в диффузионном приближении. Второй связан с решением уравнения Бибермана-Холстейна.
\end{abstract}

Ключевые слова: кинетика уровней, перенос спонтанного излучения, двухуровневая система, увеличение прозрачности среды, диффузионное приближение, уравнение Бибермана-Холстейна.

DOI: $10.21883 /$ OS.2020.08.49714.318-19

\section{Введение}

При исследовании кинетики заселенности уровней обычно используется приближение скоростных уравнений. В случае оптической накачки, к скоростным уравнениям необходимо добавить уравнения переноса излучения.

Основные процессы, определяющие заселенность уровней таковы: 1) заселение верхних уровней за счет поглощения излучения накачки и спонтанного излучения; 2) безызлучательный переход; 3) спонтанная эмиссия фотонов; 4) лазерная генерация; 5) перемешивание уровней.

В зависимости от условий нужно учитывать те или иные механизмы заселения уровней. Например, может возникнуть ситуация, когда пробеги фотонов в резонансно поглощающей среде малы по сравнению с линейными размерами среды. Это приводит к тому, что рожденный в глубине среды спонтанный фотон будет иметь высокую вероятность повторного поглощения невозбужденным атомом, и заново переизлучиться. Такой процесс будет идти до тех пор, пока спонтанно рожденный фотон не достигнет границы поглощающей среды. Происходит накопление в среде спонтанных фотонов. Это явление называют пленением излучения [1]. Данный процесс в значительной мере определяет концентрацию и пространственное распределение возбужденных атомов и тем самым влияет на кинетику заселенностей уровней. Правильное описание переноса спонтанного излучения (ПСИ) оказывается возможным лишь с помощью интегральных соотношений. Уравнение радиационного переноса впервые получено Биберманом и Холстей- ном [2-7] и носит их имена. Включение в систему кинетических уравнений уравнения Бибермана-Холстейна приводит к тому, что даже в простых задачах получение аналитического решения является проблемой невыполнимой [8]. Поэтому особое значение приобретают различные оценочные соотношения и приближенные методы.

Цель настоящей работы - исследование кинетики двухуровневой системы, с учетом ПСИ в диффузионном приближении. В этом случае удается получить аналитическое решение для двухуровневой схемы в плоском случае. Сравнение решения, полученного с помощью диффузионного приближения, с численным решением уравнения Бибермана-Холстейна позволяет оценить точность диффузионного приближения.

\section{Моделирование кинетики двухуровневой схемы с учетом переноса спонтанного излучения В диффузионном приближении}

Рассмотрим резонансное поглощение квантов накачки в двухуровневой схеме. Учтем следующие процессы: 1) заселение второго уровня фотонами накачки (фотоны, которые переводят атом из основного (невозбужденного) состояния 1 в возбужденное состояние 2,); 2) радиационный распад верхнего уровня; 3) накачка второго уровня спонтанными квантами (рис. 1). 


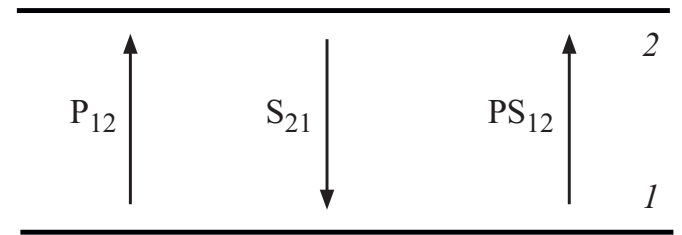

Рис. 1. Принципиальная схема двухуровневой системы. $P_{12}-$ накачка уровня $2, S_{21}-$ спонтанное излучение с уровня 2 на уровень $1, P S_{12}$ - накачка уровня 2 спонтанными фотонами.

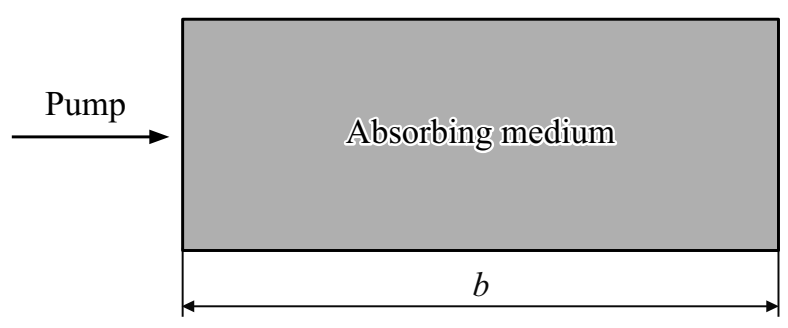

Рис. 2. Принципиальная схема задачи переноса излучения в поглощающей среде.

Система уравнений кинетики и уравнений переноса для данной задачи выглядит следующим образом [9]:

$$
\left\{\begin{array}{l}
n_{1}+n_{2}=n_{0}, \\
\frac{\partial n_{1}}{\partial t}=-\frac{1}{h v_{p}}\left(n_{1}-\frac{g_{1}}{g_{2}} n_{2}\right)\left[\sigma_{12} I_{p}(z, t)+c \sigma_{c n} U(z, t)\right]+\frac{n_{2}}{\tau}, \\
\frac{\partial n_{2}}{\partial t}=\frac{1}{h v_{p}}\left(n_{1}-\frac{g_{1}}{g_{2}} n_{2}\right)\left[\sigma_{12} I_{p}(z, t)+c \sigma_{c n} U(z, t)\right]-\frac{n_{2}}{\tau}, \\
\frac{\partial I_{p}(z, t)}{c \partial t}+\frac{\partial I_{p}(z, t)}{\partial z}=-\left(n_{1}-\frac{g_{1}}{g_{2}} n_{2}\right) \sigma_{12} I_{p}(z, t) .
\end{array}\right.
$$

Здесь $n_{1}, n_{2}$ - концентрации атомов на уровнях 1 и 2 соответственно; $\sigma_{12}$ - сечение поглощения фотона на переходе $1 \rightarrow 2 ; I_{p}(z, t)-$ интенсивность падающего излучения; $\tau$ - время жизни возбужденного атома; $n_{0}-$ концентрация атомов газа; $h v_{p}-$ энергия фотона накачки; $g_{1}, g_{2}$ - кратность вырождения энергетических уровней 1 и 2 соответственно; $c-$ скорость света; $U(z, t)$ - плотность энергии спонтанных фотонов; $\sigma_{\text {сп }}-$ сечение поглощения спонтанных фотонов.

В настоящей работе рассматривается одномерная стационарная задача (рис. 2, ось $z$ совпадает с направлением падающего излучения). Поглощающая среда имеет толщину вдоль оси $z$, равную $b$. Вдоль осей $x$ и $y$ размеры среды предполагаются бесконечно большими.

Перенос спонтанного излучения учтем в рамках диффузионного приближения, которое хорошо подходит для случая, когда пробег фотона в поглощающей среде меньше ее характерных размеров. В таком случае фотоны проходят расстояние порядка длины свободного пробега и снова поглощаются и переизлучаются. Уравнения
ПСИ в диффузионном приближении имеют вид [10]

$$
\frac{d S}{d z}=j-c \chi^{\prime} U, \mathbf{S}=-\frac{l^{\prime} c}{3} \frac{d U}{d z} \mathbf{e}_{z}, l^{\prime}=\frac{1}{\sigma_{c n}\left(n_{1}-\frac{g_{1}}{g_{2}} n_{2}\right)}
$$

Здесь $\mathbf{S}$ - вектор потока спонтанных фотонов; $l^{\prime}$ - эффективная длина пробега спонтанного фотона; $j=n_{2} h v_{p} / \tau$ - источник спонтанных фотонов.

Перейдем к безразмерным переменным

$$
\begin{gathered}
K(z)=\frac{\sigma_{12} I_{p}(z) \tau}{h v_{p}}, \quad \varphi_{1}=\frac{n_{1}}{n_{0}} \\
\varphi_{2}=\frac{n_{2}}{n_{0}}, \quad \mu=n_{0} \sigma_{12} \int_{0}^{z}\left(\varphi_{1}-\frac{g_{1}}{g_{2}} \varphi_{2}\right) d \tilde{z} \\
d \mu=n_{0} \sigma_{12}\left(\varphi_{1}-\frac{g_{1}}{g_{2}} \varphi_{2}\right) d z, \Psi(z)=\frac{c \sigma_{c n} U(z) \tau}{h v_{p}} .
\end{gathered}
$$

В системе (1) второе и третье уравнения являются линейно зависимыми. Уравнения (2) сводятся к одному уравнению. Считается, что сечение поглощения фотона накачки и спонтанного фотона одинаковы $\left(\sigma_{12}=\sigma_{c n}\right)$. Тогда в безразмерных переменных (3) система (1) совместно с уравнением ПСИ (2) примет следующий вид:

$$
\left\{\begin{array}{l}
\varphi_{1}+\varphi_{2}=1, \\
\left(\varphi_{1}-\frac{g_{1}}{g_{2}} \varphi_{2}\right) K+\left(\varphi_{1}-\frac{g_{1}}{g_{2}} \varphi_{2}\right) \Psi-\varphi_{2}=0, \\
\frac{d^{2} \Psi}{d \mu^{2}}=3 \Psi-\frac{3 \varphi_{2}}{\left(\varphi_{1}-\frac{g_{1}}{g_{2}} \varphi_{2}\right)}, \\
\frac{d K}{d z}=-n_{0} \sigma_{12}\left(\varphi_{1}-\frac{g_{1}}{g_{2}} \varphi_{2}\right) K, \\
K(0)=K_{0} .
\end{array}\right.
$$

Решением третьего уравнения системы (4) будет выражение

$$
\Psi=-3 K_{0} e^{-\mu}+C_{1} \mu+C_{2} .
$$

Для определения констант $C_{1}$ и $C_{2}$ воспользуемся граничными условиями. Поглощающая среда занимает пространство от $0 \leq z \leq b$. При $z<0$ и $z>0$ поглощающей среды нет. На границах воспользуемся условиями третьего рода [10].

Для границы слева $(z=0)$

$$
S=-\frac{c U}{2} \Rightarrow-\frac{l^{\prime} c}{3} \frac{d U}{d z}=-\frac{c U}{2} \Rightarrow\left(\frac{d \Psi}{d \mu}\right)_{\mu=0}=\frac{3}{2} \Psi_{\mu=0} .
$$

Для границы справа $(z=b)$

$$
S=\frac{c U}{2} \Rightarrow-\frac{l^{\prime} c}{3} \frac{d U}{d z}=\frac{c U}{2} \Rightarrow\left(\frac{d \Psi}{d \mu}\right)_{\mu=\mu_{0}}=-\frac{3}{2} \Psi_{\mu=\mu_{0}},
$$




$$
\mu_{0}=n_{0} \sigma_{12} \int_{0}^{b}\left(\varphi_{1}-\frac{g_{1}}{g_{2}} \varphi_{2}\right) d z .
$$

Из условий (6) и (7) выражаем константы $C_{1}$ и $C_{2}$. Зависимость $\varphi_{1}=\varphi_{1}(\mu)$ имеет следующий вид:

$$
\varphi_{1}=\frac{1+\frac{g_{1}}{g_{2}}\left(C_{1} \mu+C_{2}-2 K_{0} e^{-\mu}\right)}{1+\left(1+\frac{g_{1}}{g_{2}}\right)\left[C_{1} \mu+C_{2}-2 K_{0} e^{-\mu}\right]} .
$$

Зависимость $z=z(\mu)$ определяется из следующего уравнения:

$$
\frac{d \mu}{d z}=n_{0} \sigma_{12}\left(\varphi_{1}-\frac{g_{1}}{g_{2}} \varphi_{2}\right) .
$$

Так как $\mu \rightarrow 0$ при $z \rightarrow 0$, получаем

$n_{0} \sigma_{12} z=\mu+\left(1+\frac{g_{1}}{g_{2}}\right)\left(2 K_{0} e^{-\mu}-2 K_{0}+\frac{C_{1} \mu^{2}}{2}+C_{2} \mu\right)$.

Оптическую толщину $\mu_{0}$ получим из уравнения (8).

В итоге имеем параметрическую зависимость всех переменных от числа $\mu$.

Учет переноса спонтанных фотонов приводит к увеличению прозрачности поглощающей среды

$$
\chi^{\prime}=\sigma_{12}\left(n_{1}-\frac{g_{1}}{g_{2}} n_{2}\right)=\frac{n_{0} \sigma_{12}}{1+\frac{\sigma_{12} \tau}{h_{p}}\left(1+\frac{g_{1}}{g_{2}}\right)\left(I_{p}+c U\right)} .
$$

Здесь $\chi^{\prime}-$ коэффициент поглощения. Из формулы (9) видно, что коэффициент поглощения зависит от интенсивности падающего излучения и плотности энергии спонтанных квантов. Это значит, что с ростом интенсивности падающего излучения коэффициент поглощения будет становиться меньше, т.е. среда увеличивает прозрачность.

\section{Моделирование кинетики двухуровневой схемы с учетом ПСИ с использованием уравнения Бибермана-Холстейна}

Рассмотрим такую же задачу, что и выше, для случая, когда ПСИ и его вклад в кинетику описывается уравнением Бибермана-Холстейна [11], которое в случае резонансного поглощения имеет вид

$$
\frac{\partial n}{\partial t}=\frac{1}{\tau} \int_{V} n\left(r^{\prime}\right) \frac{\chi^{\prime}}{4 \pi\left|\mathbf{r}-\mathbf{r}^{\prime}\right|^{2}} \exp \left(-\int_{\mathbf{r}^{\prime}}^{\mathbf{r}} \chi^{\prime} d x\right) d V .
$$

Здесь $d V$ - объем элементарной ячейки среды, которая является источником спонтанных квантов; $\chi^{\prime}-$ коэффициент поглощения; $\mathbf{r}-$ координата точки, в которой рассчитывается кинетика; $\mathbf{r}^{\prime}-$ координата объема $d V$; $\exp \left(-\int \chi^{\prime} d x\right)-$ представляет долю дошедшего из точки $\mathbf{r}^{\prime}$ в точку $\mathbf{r}$ излучения.

Выражение (10) преобразуем к простому виду. В направлениях $x$ и $y$ среда является бесконечной, значит,
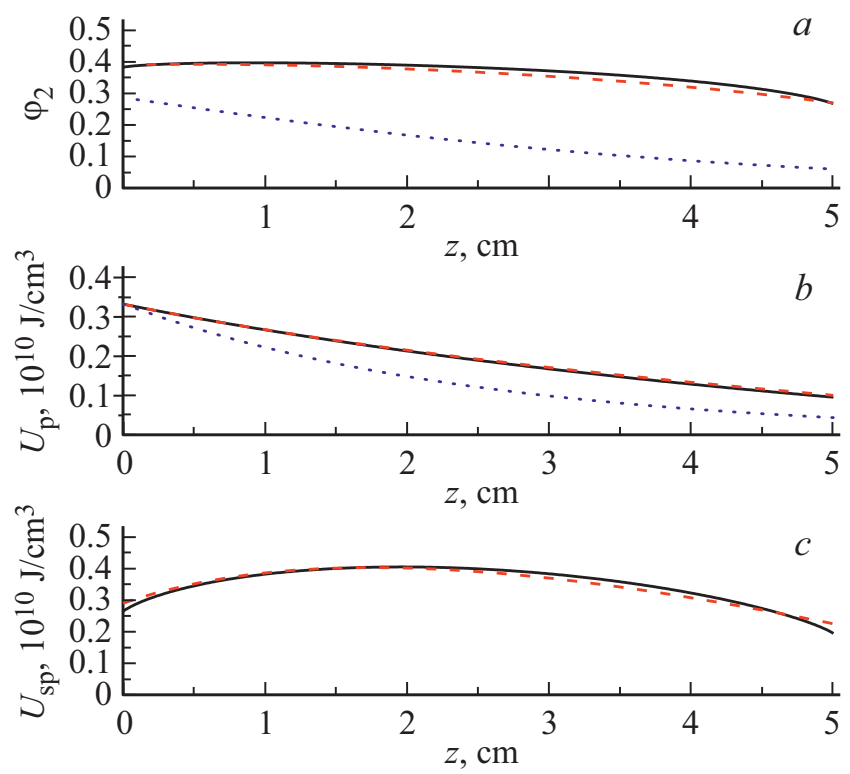

Рис. 3. (a) Распределение концентрации атомов в возбужденном состоянии; $(b)$ плотность энергии накачки; $(c)$ плотность энергии спонтанного излучения в зависимости от глубины проникновения накачки.

относительно оси $z$ задача обладает симметрией. Следовательно, элементарный объем $d V$ можно выразить через объем кольца:

$$
d V=2 \pi \rho d \rho d z^{\prime}=\frac{\left(z-z^{\prime}\right)^{2}}{\cos ^{2}(\theta)} \operatorname{tg}(\theta) d \theta d z^{\prime}
$$

Уравнения кинетики совместно с уравнением переноса двухуровневой схемы в безразмерных переменных имеют вид

$$
\left\{\begin{array}{l}
\varphi_{1}+\varphi_{2}=1 \\
\frac{\partial \varphi_{2}}{\partial(t / \tau)}=\left(\varphi_{1}-\frac{g_{1}}{g_{2}} \varphi_{2}\right) K(z)+\frac{\chi^{\prime}}{2}\left[F_{1}(z)+F_{2}(z)\right] \\
F_{1}(z)=n_{0} \int_{0}^{z} \varphi_{2}\left(z^{\prime}\right) E_{1}\left(\int_{z^{\prime}}^{z} \chi^{\prime}\left(z^{\prime \prime}\right) d z^{\prime \prime}\right) d z^{\prime} \\
F_{2}(z)=n_{0} \int_{z}^{b} \varphi_{2}\left(z^{\prime}\right) E_{1}\left(\int_{z}^{z^{\prime}} \chi^{\prime}\left(z^{\prime \prime}\right) d z^{\prime \prime}\right) d z^{\prime} \\
K(z)=K_{0} \exp (-\mu(z)) .
\end{array}\right.
$$

Функция $F_{1}$ отвечает за спонтанные фотоны, которые прибывают в точку $z$ из левой части пространства, а функция $F_{2}$ отвечает за спонтанные фотоны, которые прибывают в точку $z$ из правой части пространства (рис. 3), $E_{1}$ - интегральная экспонента. 
Систему (11) можно свести к интегродифференциальному уравнению

$$
\begin{aligned}
\frac{\partial \varphi_{2}}{\partial(t / \tau)} & =\left(1-\varphi_{2}-\frac{g_{1}}{g_{2}} \varphi_{2}\right) \\
& \times\left[K(z)+\frac{\sigma_{12}}{2} F_{1}(z)+\frac{\sigma_{12}}{2} F_{2}(z)\right]-\varphi_{2} .
\end{aligned}
$$

Уравнение (12) решаем численным методом. Поглощающая среда по направлению $z$ разбивается на $N$ слоев, каждый толщиной $b / N$. Тогда уравнение (12) разобьется на $N$ дифференциальных уравнений, каждое из которых описывает временную эволюцию концентрации на втором уровне в своей точке пространства. При численном счете рассматривается именно нестационарная задача. Расчет продолжается до некоторого характерного времени, после которого все параметры задачи перестают зависеть от времени (т.е. принимают стационарные значения). Характерное время определяется из графиков, в которых концентрация, зависящая от времени, выходит на постоянное значение.

\section{Сравнение диффузионного приближения с приближением Бибермана-Холстейна}

Анализ получившихся выше решений, проводится на примере двухуровневой газовой среды, состоящей из паров Na. Выбор Na связан с тем, что этот атом достаточно хорошо изучен (известны все необходимые для расчета константы [12]). Также Na можно рассматривать как двухуровневую систему, так как при концентрации атомов (натрия) $n_{0} \sim 10^{11} \mathrm{~cm}^{-3}$ и при температуре $T=3.45 \cdot 10^{-2} \mathrm{eV}$ сечение поглощения излучения перехода $3 S_{1 / 2} \rightarrow 3 P_{3 / 2}$ порядка $10^{-11} \mathrm{~cm}^{2}$, что много больше сечения перемешивания уровней тонкой структуры уровня $3 P$, которое порядка $10^{-17} \mathrm{~cm}^{2}$. Это означает, что заселенность уровня $3 P_{1 / 2}$ будет ничтожна мала (по сравнению с заселенностью на уровнях $3 S_{1 / 2}$ и $\left.3 P_{3 / 2}\right)$, и он не будет участвовать в кинетике.

Рассматривается переход валентного электрона из основного состояния $3 S_{1 / 2}$ в состояние $3 P_{3 / 2}$. Энергетическое расстояние между данными уровнями $E=2.1 \mathrm{eV}$. Время жизни уровня $3 P_{3 / 2}$ составляет $\tau=16.25 \mathrm{~ns}$. Масса атома Na равна $m_{\mathrm{Na}} c^{2}=21.63 \mathrm{GeV}$. Длина волны перехода $3 S_{1 / 2} \rightarrow 3 P_{3 / 2}$ равна $\lambda_{0}=589 \mathrm{~nm}$.

В данной постановке можно считать, что спектральная линия имеет допплеровскую форму:

$$
a_{\omega}=\frac{1}{\omega_{0}}\left(\frac{m_{\mathrm{Na}} c^{2}}{2 \pi T}\right)^{1 / 2} \exp \left[-\frac{m_{\mathrm{Na}} c^{2}\left(\omega-\omega_{0}\right)^{2}}{2 \omega_{0}^{2} T}\right] .
$$

Здесь $\omega_{0}=2 \pi c / \lambda_{0}, \lambda_{0}$ - длина волны света в максимуме линии.
Сечение поглощения перехода $3 S_{1 / 2}$ в $3 P_{3 / 2}$ рассчитывается по формуле [11]

$$
\sigma_{S_{1 / 2} \rightarrow P_{3 / 2}}=\left(\frac{\pi c}{\omega}\right)^{2} \frac{a_{\omega}}{\tau} \frac{g_{P_{3 / 2}}}{g_{S_{1 / 2}}} .
$$

Здесь $g_{P_{3 / 2}}-$ кратность вырождения уровня $3 P_{3 / 2}$, равная $4, g_{S_{1 / 2}}-$ кратность вырождения уровня $3 S_{1 / 2}$, равная 2.

При резонансном поглощении фотонов из формул (13) и (14) получается, что $\sigma_{P_{3 / 2} \rightarrow S_{1 / 2}}=$ $=1.07 \cdot 10^{-11} \mathrm{~cm}^{2}$.

Приведем пример для интенсивности накачки $I_{p}(0)=1 \mathrm{~W} / \mathrm{cm}^{2}$. Концентрация атомов $\mathrm{Na}$ равна $n_{0}=5 \cdot 10^{10} \mathrm{~cm}^{-3}$. Толщину области, заполненную поглощающим газом, примем равной $b=5 \mathrm{~cm}$. На рис. $3, a, b$ представлены три кривые. Штриховая кривая соответствует аналитическому решению задачи (4). Сплошная кривая соответствует численному решению уравнения (12). Точечная кривая соответствует решению, в котором не учитывается ПСИ. На рис. $3, c$ изображены распределения плотности спонтанного излучения вдоль координаты $z$ для двух приближений. Из этих графиков видно, что оба приближения при заданных параметрах имеют удовлетворительное согласие.

Как видно из результатов, приведенных на рис. $3, a, b$, вклад спонтанных фотонов в накачку среды является важным. На рис. 3, $c$ представлена зависимость плотности спонтанного излучения. Это и есть те самые „плененные“ спонтанные кванты, которые из-за малых пробегов (по сравнению с размерами среды) покидают среду не сразу. Сравнивая рис. $3, b$ и $3, c$, можно заметить, что плотность спонтанного излучения превосходит плотность энергии накачки, что приводит к дополнительной накачке среды. Численный эксперимент показывает, что если понизить концентрацию поглощающих центров (атомов натрия), то тем самым увеличится длина свободного пробега в среде и вклад в кинетику спонтанных квантов станет намного меньше, чем вклад квантов накачки. Увеличение интенсивности накачки приводит к увеличению прозрачности вещества (длина свободного пробега фотона увеличится). Спонтанные фотоны будут легко покидать поглощающую среду, что приведет к уменьшению их вклада в кинетику среды. Таким образом, можно заключить, что параметром, который определяет вклад спонтанного излучения в кинетику, является длина свободного пробега.

На рис. 4 представлено решение уравнения (12). Это уравнение, как сказано выше, решалось численным методом. Поглощающая среда разбивалась по толщине на $N$ слоев. Для каждого слоя (каждый имеет толщину $b / N)$ получалось свое уравнение, которое описывало временную эволюцию концентрации атомов в возбужденном состоянии. Несмотря на то, что уравнение (12) решалось без учета временной производной в уравнении 


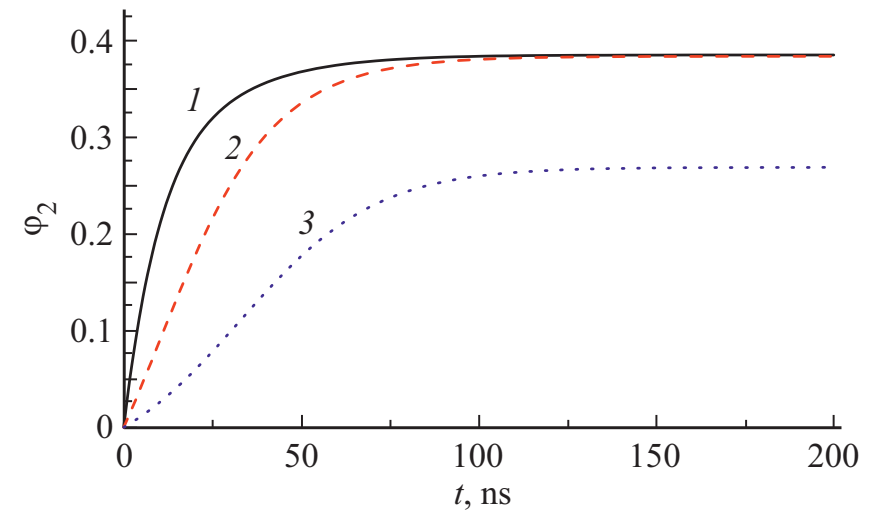

Рис. 4. Зависимость концентрации возбужденных атомов от времени. Кривая 1 соответствует изменению концентрации в первом слое $(z=0)$, кривая 2 соответствует серединному слою $(z=b / 2)$, кривая 3 соответствует последнему слою $(z=b)$.

переноса излучения накачки, в результате предварительного расчета получено, что интенсивность излучения накачки выходит на стационарное значение через время $\sim b / c(c-$ скорость света). Для рассматриваемого примера это время $\approx 0.17 \mathrm{~ns}$.

Однако из рис. 4 видно, что время, через которое в среде устанавливается стационарное значение концентрации возбужденных атомов, много больше времени установления стационарной интенсивности излучения накачки. Следовательно, зависимостью интенсивности излучения накачки от времени можно пренебречь, и время, через которое уравнение (12) становиться стационарным, равно времени, через которое в среде установится концентрация атомов в возбужденном состоянии.

Из рис. 4 видно, что приблизительно через время, равное 120 ns, во всех слоях концентрация (следовательно, и все остальные величины) примет свое стационарное значение. Именно эти стационарные значения и рассматривались в настоящей работе и сравнивались со значениями, получаемыми в диффузионном приближении.

\section{Заключение}

Получено аналитическое решение задачи о заселение двух уровней с учетом ПСИ в диффузионном приближении.

Решение этой же задачи получено с помощью численного расчета интегро-дифференциального уравнения, в котором ПСИ описывался уравнением Бибермана-Холстейна.

Получено, что оба подхода удовлетворительно согласуются в диапазоне оптических толщин от 0 до $\sim 10^{1}-10^{2}$. Показано (рис. $3, a, b, c$ ), что в случае, когда характерные размеры среды сравнимы или больше эффективной длины свободного пробега фотона в этой среде, заметный вклад в кинетику уровней вносит спонтанное излучение. При понижении концентрации поглощающих центров (атомов $\mathrm{Na}$ ) или при повышении потока излучения накачки происходит увеличение эффективной длины свободного пробега фотона и уменьшение концентрации спонтанных фотонов в среде (исчезает эффект пленения излучения).

\section{Конфликт интересов}

Авторы заявляют, что у них нет конфликта интересов.

\section{Список литературы}

[1] Голубовский Ю.Б., Некучаев В.О., Сясько А.В. // Хим. физика. 2015. Т. 34. № 8. С. 29.

[2] Holstein T. // Phys. Rev. 1947. N 72. P. 1212.

[3] Биберман Л.М. // ЖЭТФ. 1947. Т. 17. С. 416.

[4] Преображсенский Н.Г., Суворов А.Е. // ПТМФ. 1977. № 2. C. 3.

[5] Косарев Н.И. // Матем. моделирование. 2008. Т. 20. № 3. C. 87.

[6] Биберман Л.М., Воробьев В.С., Якубов И.Т. Кинетика неравновесной низкотемпературной плазмы. М.: Наука, 1982. $374 \mathrm{c}$.

[7] Huennekens J., Park H.J., Colbert T., McClain S.C. // Phys. Rev. 1987. N 3. P. 35.

[8] Нагирнер Д.И. Лекции по теории переноса излучения: Учеб. пособие. СПб.: Изд-во СПбУ, 2001. 147 с.

[9] Тарасов Л.В. Физика процессов в генераторах когерентного оптического излучения. М.: Радио и связь, 1981. 440 с.

[10] Зельдович Я.Б., Райзер Ю.П. Физика ударных волн и высокотемпературных гидродинамических явлений. 3-е изд., исправл. М.: Физматлит, 2008. 656 с.

[11] Крайнов В.П., Смирнов Б.М. Квантовая теория излучения атомных частиц: Учеб. пособие Долгопрудный: Издательский дом „Интеллект“, 2015. 296 с.

[12] Daniel A. Steck Sodium D Line Data.[Электронный pecypc] Режим доступа: http://steck.us/alkalidata 